\title{
Expressing a cytosolic pyruvate dehydrogenase complex to increase free fatty acid production in Saccharomyces cerevisiae
}

\author{
Yiming Zhang ${ }^{1 \dagger}$, Mo Su$^{1 \dagger}$, Ning Qin ${ }^{1}$, Jens Nielsen ${ }^{1,2,3}$ and Zihe Liu ${ }^{1 *}$ (D)
}

\begin{abstract}
Background: Saccharomyces cerevisiae is being exploited as a cell factory to produce fatty acids and their derivatives as biofuels. Previous studies found that both precursor supply and fatty acid metabolism deregulation are essential for enhanced fatty acid synthesis. A bacterial pyruvate dehydrogenase (PDH) complex expressed in the yeast cytosol was reported to enable production of cytosolic acetyl-CoA with lower energy cost and no toxic intermediate.

Results: Overexpression of the PDH complex significantly increased cell growth, ethanol consumption and reduced glycerol accumulation. Furthermore, to optimize the redox imbalance in production of fatty acids from glucose, two endogenous $\mathrm{NAD}^{+}$-dependent glycerol-3-phosphate dehydrogenases were deleted, and a heterologous $\mathrm{NADP}^{+}$-dependent glyceraldehyde-3-phosphate dehydrogenase was introduced. The best fatty acid producing strain PDH7 with engineering of precursor and co-factor metabolism could produce $840.5 \mathrm{mg} / \mathrm{L}$ free fatty acids (FFAs) in shake flask, which was $83.2 \%$ higher than the control strain YJZ08. Profile analysis of free fatty acid suggested the cytosolic PDH complex mainly resulted in the increases of unsaturated fatty acids (C16:1 and C18:1).
\end{abstract}

Conclusions: We demonstrated that cytosolic PDH pathway enabled more efficient acetyl-CoA provision with the lower ATP cost, and improved FFA production. Together with engineering of the redox factor rebalance, the cytosolic PDH pathway could achieve high level of FFA production at similar levels of other best acetyl-CoA producing pathways.

Keywords: The pyruvate dehydrogenase complex, Free fatty acids, Redox factor, Saccharomyces cerevisiae

\section{Background}

Bioproduction of fatty acids and their derivatives serves as a sustainable route to alleviate the dilemma between the growing demands of high-energy-density fuels and concerns on their environmental impacts. However, fatty acids are involved in several essential cellular processes, such as membrane synthesis, energy storage, and protein

\footnotetext{
${ }^{*}$ Correspondence: zihe@mail.buct.edu.cn

†Yiming Zhang and Mo Su contributed equally to this work

${ }^{1}$ Beijing Advanced Innovation Center for Soft Matter Science and Engineering, College of Life Science and Technology, Beijing

University of Chemical Technology, No.15 North Third Ring Road East, Chaoyang District, Beijing 100029, People's Republic of China

Full list of author information is available at the end of the article
}

modification, and their levels are therefore tightly regulated to ensure in vivo homeostasis [1]. Intensive efforts have been carried out to improve the titer, rate and yield (TRY) of fatty acid-derived biofuels, focusing mainly on the deregulation of fatty acid metabolism and redirection of carbon flux [2-4].

Regarding deregulation of fatty acid metabolism, deletions of dominant acyl-CoA synthetases (FAA1, FAA4), and fatty acyl-CoA oxidase (POX1) catalyzing the first step in $\beta$-oxidation, could significantly accumulate free fatty acids (FFAs) [5-7]. Moreover, deletions of main sterol ester formation genes (ARE1, ARE2), and phosphatidate phosphatase genes (PAH1, DPP1, LPP1) could also deregulate fatty acid synthesis and increase the 
production of total fatty acids [8]. Combining deletion of several lipid regulation mechanisms resulted in a reduced complexity of the fatty acid metabolic network, leading to enhanced FFA accumulation $[8,9]$.

Besides engineering lipid metabolic network, engineering of precursor supply and redox cofactor regeneration was also essential for carbon flux redirection towards FFA production. Each elongation cycle in fatty acid synthesis extends the backbone with a $\mathrm{C} 2$ unit and requires 2 molecules of NADPH as cofactor. Endogenous synthesis of cytosolic acetyl-CoA, the main $\mathrm{C} 2$ metabolite, is often limited and several heterologous enzymes have therefore been expressed to increase acetyl-CoA supply for FFA synthesis, including $\mathrm{NAD}^{+}$-dependent alcohol dehydrogenase (AdhE), $\mathrm{NAD}^{+}$-dependent acylating acetaldehyde dehydrogenase (EutE) [10], ATP citrate lyases (ACLs) [6, 7, 11-13], xylulose-5-phosphate phosphoketolase (XpkA) and phosphotransacetylase (Pta) [14]. Compared to these strategies, expression of cytosolic pyruvate dehydrogenase (PDH) is attractive because of its lower energy cost [15]. The PDH complex consists of three catalytic subunits, including pyruvate dehydrogenase (E1), dihydrolipoyl transacetylase (E2), and dihydrolipoyl dehydrogenase (E3), and requires four kinds of cofactors TPP, lipoate, FAD and $\mathrm{NAD}^{+}$. Among three $\mathrm{PDH}$ complexes that have been expressed in the cytosol of yeast, PDH from Enterococcus faecalis was demonstrated to enable complete replacement of the native pathway for cytosolic acetyl-CoA synthesis, and was less sensitive to high $\mathrm{NADH} / \mathrm{NAD}^{+}$ratios compared with other PDH complexes [16-18]. However, one concern with PDH expression for FFA production is the redox imbalance between redox couples of $\mathrm{NAD}^{+} / \mathrm{NADH}$ and $\mathrm{NADP}^{+} / \mathrm{NADPH}$.
Previous studies showed that high glycerol production was often observed in FFA production strains, indicating a high NADH pressure [7]. Glycerol synthesis from dihydroxyacetone phosphate consists of two steps, catalyzed by $\mathrm{NAD}^{+}$-dependent glycerol-3-phosphate dehydrogenase (encoded by GPD1 and GPD2) and glycerol-3-phosphate phosphatase (encoded by GPP1 and GPP2), respectively (Fig. 1). Glycerol accumulation is also an issue in bioethanol production and has therefore been studied for years. Several studies found that glycerol formation could be decreased with altered redox-cofactor specificity [11, 19-22], and could be eliminated through inactivation of both GPD1 and GPD2 with the expense of a dramatic reduction in cell growth [23]. As alternatives, an $\mathrm{NADP}^{+}$-dependent glyceraldehyde-3-phosphate dehydrogenase $(\mathrm{GapN})$ was demonstrated to enable rescuing the negative effects and improve ethanol yield [24, 25]. Moreover, since GapN irreversibly converts glyceraldehyde-3-phosphate to 3-phosphoglycerate and produces NADPH (Fig. 1), it was reported to increase the synthesis of NADPH demanding bioproducts, including 3-hydroxypropionic acid (3-HP) and polyhydroxybutyrate (PHB) $[26,27]$. Therefore, it could potentially eliminate glycerol accumulation and increase FFA production by interruption of glycerol synthesis and expression of GapN.

The goal of this study is to investigate the effect of expressing the cytosolic PDH complex on FFA production in yeast strains with deregulated fatty acid metabolism and optimized cytosolic redox balance (Fig. 1). The strategies reported here may shed lights for production of other acetyl-CoA and NADPH demanding chemicals as well.

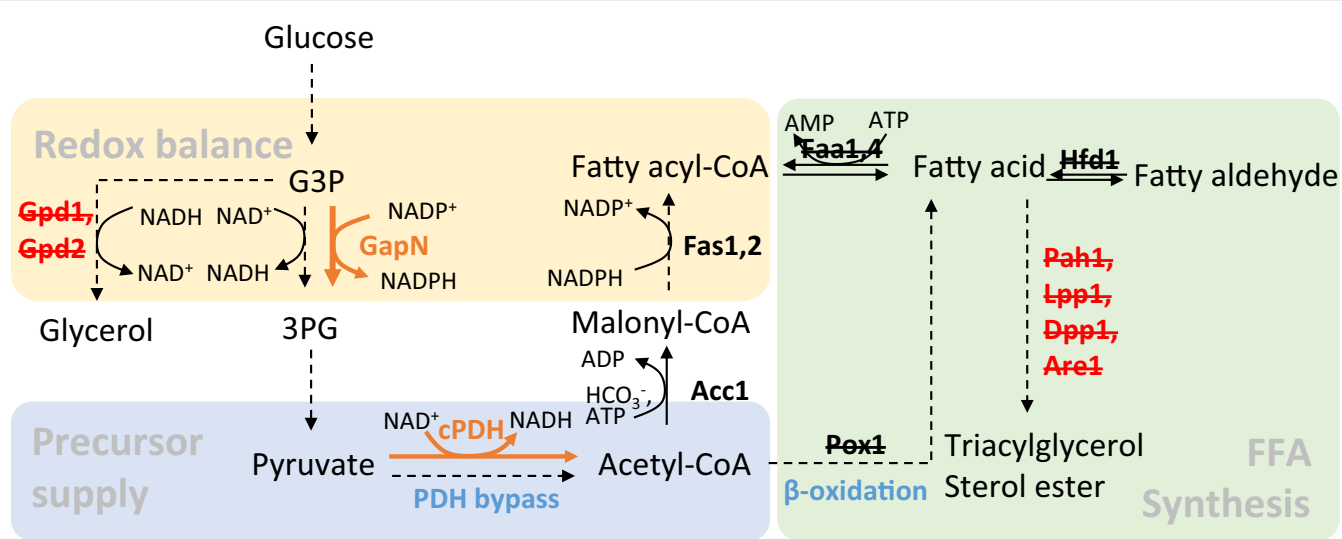

Fig. 1 Schematic representation of engineering strategies for FFA production in S. cerevisiae. G3P glyceraldehyde-3-phosphate, 3PG 3-phospho-glycerate, CPDH cytosolic pyruvate dehydrogenase. A cytosolic PDH complex was evaluated in a faa $1 \triangle$ faa4 $\Delta$ pox $1 \Delta$ hfd1 $\triangle$ mutant YJZ08 for free fatty acid production, in combination with fatty acid metabolism engineering via pah $1 \Delta$ lpp $1 \Delta d p p 1 \Delta$ are $1 \Delta$ and redox cofactor engineering via GPD deletion and GapN expression 


\section{Results and discussion}

\section{Expression of a bacterial PDH complex to increase the acetyl-CoA supply}

A FFA producing strain YJZ08 with four gene deletions (faa1 $\operatorname{faa} 4 \Delta$ pox $1 \Delta$ hfd1 $)$ was used as the background strain for cytosolic PDH evaluation [6]. The three subunits of PDH complex, encoded by $p d h A, p d h B$, aceF and $l p d$, and lipoate ligases from $E$. faecalis, encoded by $l p l A$ and lplA2 [16], were integrated into the two genome sites Chr XII-5 and Chr XI-3 [28] of, yielding strain PDH1. As shown in Fig. 2, YJZ08 with deregulated FA synthesis produced FFA at $458.9 \mathrm{mg} / \mathrm{L}$ in $72 \mathrm{~h}$ in minimal medium with $20 \mathrm{~g} / \mathrm{L}$ glucose, which was consistent with previous studies [6, 9]. Meanwhile, the constructed strain PDH1 produced $512.7 \mathrm{mg} / \mathrm{L}$ FFA at $72 \mathrm{~h}$ (Fig. 2A) with a lower ethanol yield (Additional file: Table S1). From the growth data, we found that $E$. faecalis $\mathrm{PDH}$ reduced the maximum specific growth rate from $0.33 \mathrm{~h}^{-1}$ to $0.28 \mathrm{~h}^{-1}$ but didn't result in much difference in the final biomass. PDH1 accumulated $1.90 \mathrm{~g} / \mathrm{L}$ glycerol, compared with
1.90 g/L glycerol with YJZ08 (Fig. 2a), suggesting that cytosolic PDH did not cause an NADH burden in YJZ08.

Theoretically, a combination of the $\mathrm{PDH}$ route with the phosphoketolase $(\mathrm{PK})$ pathway can result in a higher maximum FFA yield [15], since the PDH route could produce acetyl-CoA with low energy cost and the PK pathway could produce acetyl-CoA with low carbon loss. Moreover, the PK pathway utilizes xylulose-5-phosphate from the pentose phosphate (PP) pathway as the substrate, and it may thermodynamically help to pull the carbon flux towards the PP pathway and increase NADPH generation. However, we tested the integration of xylulose-5-phosphate phosphoketolase from Leuconostoc mesenteroides [29] and phosphotransacetylase from Clostridium kluyveri [30] into both YJZ08 and PDH1, and did not find significant improvement in cell growth and FFA synthesis (Additional file 1: Figure S1), probably because of the strict control of carbon flux through the PP pathway [31] or inefficient phosphoketolase enzymes [29].

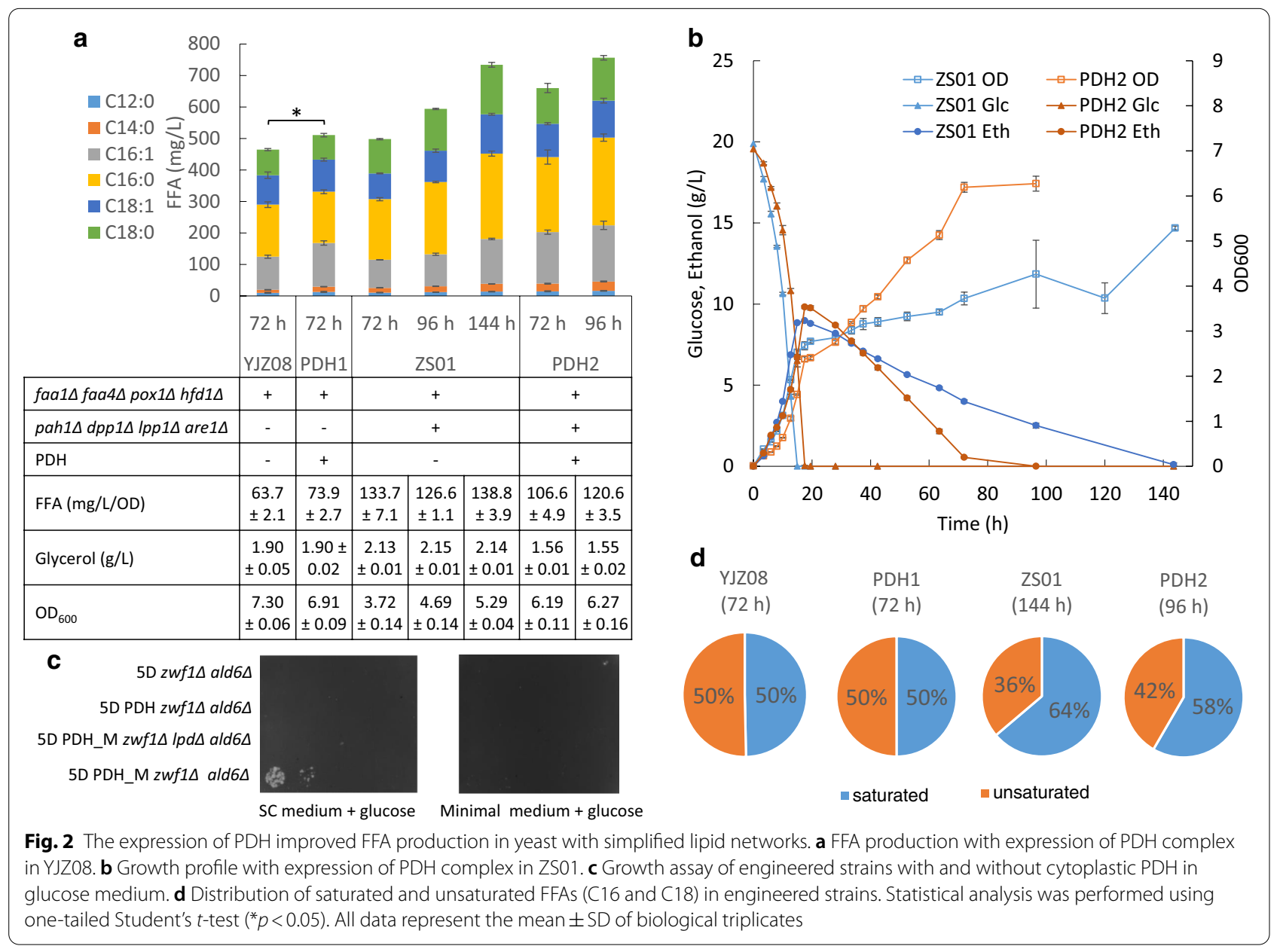


Moreover, the yeast ZS01 strain with simplified lipid metabolic networks and redirected flux towards FFA synthesis was constructed by deletion of PAH1, DPP1, LPP1 and $A R E 1$ in YJZ08. Then, the genes encoding the PDH complex, composing of $p d h A, p d h B, a c e F, l p d, l p l A$ and lplA2, were integrated into the chromosome of ZS01, yielding strain PDH2. As shown in Fig. 2, when cultivated in minimal medium with $20 \mathrm{~g} / \mathrm{L}$ glucose, ZS01 accumulated $497.9 \mathrm{mg} / \mathrm{L}$ FFAs at $72 \mathrm{~h}$ with a high productivity of $133.7 \mathrm{mg} / \mathrm{L} / \mathrm{OD}$, which was consistent with previous studies [8, 9]. After $72 \mathrm{~h}, \mathrm{ZS} 01$ accumulated more FFAs since there was ethanol remaining and the FFA titer reached $734.2 \mathrm{mg} / \mathrm{L}$ at $144 \mathrm{~h}$ when ethanol was exhausted (Fig. 2a). Interestingly, PDH2 produced $660.2 \mathrm{mg} / \mathrm{L}$ FFAs at $72 \mathrm{~h}$ and $756.3 \mathrm{mg} / \mathrm{L}$ FFAs at $96 \mathrm{~h}$ when ethanol was completely exhausted. In PDH2, the faster FFA production rate accompanied with a faster ethanol consumption rate and lower glycerol level, indicating that cytosolic $\mathrm{PDH}$ may alleviate NADH burden and speed up cell growth and FFA production (Fig. $2 \mathrm{~b}$ ), probably due to the lower energy cost.

To address the possible mismatch between acetylCoA supply, NADH production and NADPH consumption for FFA synthesis, we evaluated whether PDH from E. faecalis might utilize $\mathrm{NADP}^{+}$as well as $\mathrm{NAD}^{+}$as redox cofactors in a $z w f 1 \Delta$ ald $6 \Delta$ mutant, which hardly grew on glucose medium due to lack of ability to produce cytosolic NADPH [32]. The results showed that cytosolic PDH expression could not rescue cell growth of the $z w f 1 \Delta$ ald $6 \Delta$ mutant on glucose (Fig. 2c). A previous study showed that cofactor specificity of $E$. coli $\mathrm{PDH}$ could be converted from $\mathrm{NAD}^{+}$to $\mathrm{NADP}^{+}$via seven amino acid mutations in E3 [33, 34]. The alignment result of E3 proteins from E. faecalis and E. coli suggested that the mutated amino acids are highly conserved, as shown in Additional file 1: Figure S2a. Expression of the mutated PDH recued the growth on glucose and disruption of E3 encoding gene lpd suspended the growth recovery, suggesting the mutated PDH might utilize $\mathrm{NADP}^{+}$as redox cofactor. Growth assay on minimal glucose medium didn't show similar results, which might be caused by its poor activity (Fig. 2c). However, these E3 mutations didn't increase FFA titer, but reduced the titer to $439.5 \mathrm{mg} / \mathrm{L}$, comparable with YJZ08 (Additional file 1: Figure S2b and Table S1). The FFA results indicated that the mutations resulted in enzymatic activity lost, probably due to unsuccessful protein assembly. To improve its enzymatic activity towards $\mathrm{NADP}^{+}$, rational design based on PDH structures or directed evolution will be required for further investigation. As E. faecalis PDH has been demonstrated to be a functional enzyme in $E$. coli and yeast at both aerobic and anaerobic conditions $[16,35]$, a novel PDH complex with $\mathrm{NADP}^{+}$preference will clearly be valuable for bioproduction of acetyl-CoA derived chemicals.

It was also observed that, PDH expression altered the distribution of saturated and unsaturated FFAs (C16 and C18) in PDH2, but not in PDH1 (Fig. 2d). The double bonds in unsaturated FFAs were formed by Delta- 9 fatty acid desaturase, encoded by OLE1, through an oxygendependent mechanism that requires reducing equivalents from NADH. Thus, the increase in unsaturated FFA ratio might also indicate that the functional PDH resulted in cytosolic redox changes without imposing an increased NADH burden, due to its insensitive feature to high NADH/NAD ${ }^{+}$ratios [35-37].

\section{Balance of cytosolic redox factors for FFA production}

NAD and NADP redox couples are crucial to maintain cellular redox hemostasis, with lower cytosolic NADH/ $\mathrm{NAD}^{+}$ratios and higher NADPH/NADP ${ }^{+}$ratios due to their different functions [38]. In FFA production strains, over-production of NADH resulted in glycerol accumulation, while NADPH as the cofactor required for fatty acid elongation was limited. Thus, the balance between NAD and NADP redox couples would be important for FFA production.

To balance the redox state of the cell and further improve FFA synthesis, $\mathrm{NADP}^{+}$-dependent glyceraldehyde-3-phosphate dehydrogenase $(G a p N)$ from Streptococcus mutans [39] was expressed on a multi-copy plasmid pGapN in the FFA production strain YJZ08, yielding strain ZS02. As shown in Fig. 3, GapN expression resulted in reduced glycerol accumulation and increased FFA production.

We further balanced cytosolic redox conditions through interruption of glycerol synthesis via glycerol3-phosphate dehydrogenase (GPD) deletions. Since GPD1 was regulated by osmotic stress [23], GPD2 was first deleted in YJZ08, and the resulting strain ZS03 showed decreased glycerol accumulation, and increased FFA titer and productivity (Fig. 3a). When both GPD1 and GPD2 were deleted, the specific growth rate of the resulted strain ZS04 decreased dramatically, which was consistent with previous studies [23]. The final FFA titer of ZS03 was $556.0 \mathrm{mg} / \mathrm{L}, 23.6 \%$ higher than YZJ08. When GapN was introduced into ZS04, the impaired growth was improved, and FFA production at $72 \mathrm{~h}$ improved significantly. The final FFA titer of ZS05 reached $578.7 \mathrm{mg} / \mathrm{L}$ at $96 \mathrm{~h}$, which was $28.6 \%$ higher than YJZ08 and the productivity was comparable with ZSO4.

Similarly, the distributions of saturated and unsaturated FFA also varied in the constructed strains (Fig. 3b). GPD deletion did not alter the distributions, whereas GapN expression increased the ratios of saturated FFAs. With GapN expressed in YJZ08, the percentage of saturated 


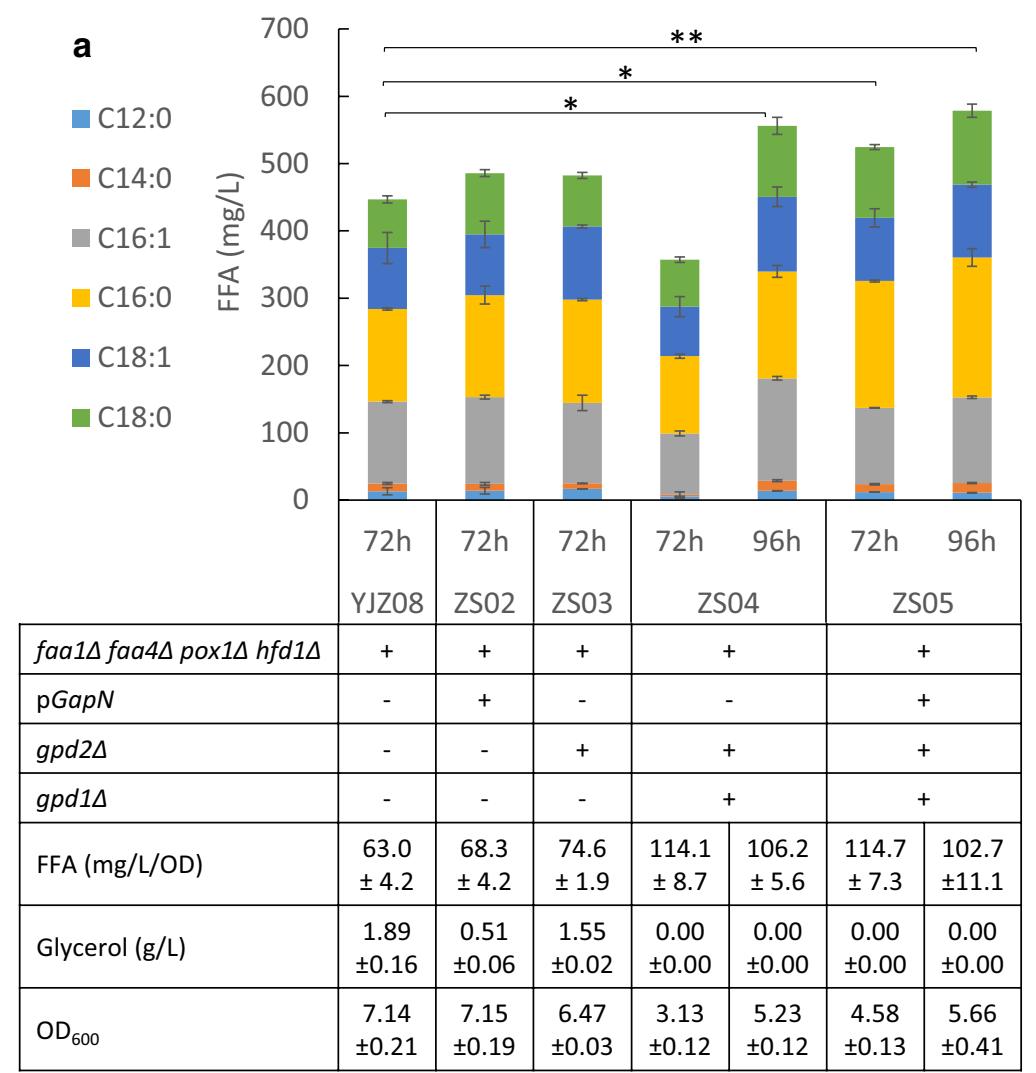

b
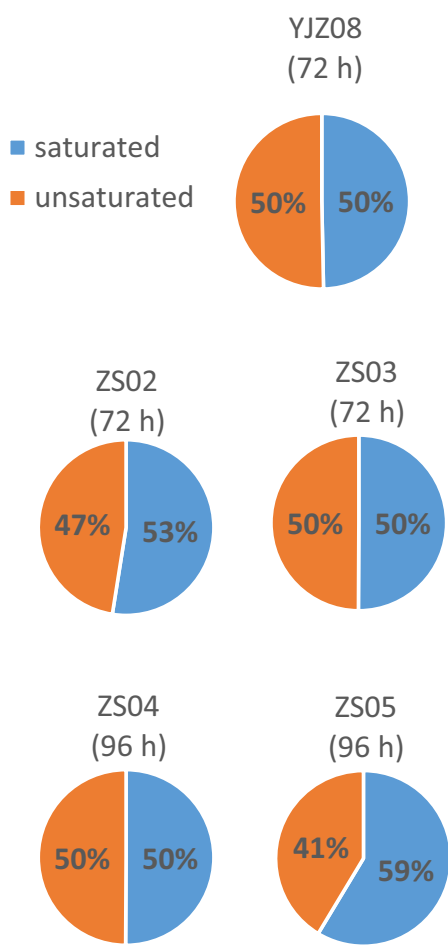

ZS05

$(96 \mathrm{~h})$

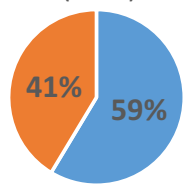

Fig. 3 FFA production with GapN expression and GPD deletions. a FFA production obtained with engineered strains. b Distribution of saturated and unsaturated FFAs (C16 and C18) in the engineered strains. Statistical analysis was performed using one-tailed Student's $t$ test $\left(^{*} p<0.05\right.$, $\left.{ }^{* *} p<0.01\right)$. All data represent the mean \pm SD of biological triplicates

FFAs increased slightly from 50 to $53 \%$, with the increases in the titers of C16:0 and C18:0 but no increases of C16:1 and C18:1. When GapN expressed in gpd1 $\operatorname{gpd} 2 \Delta$ mutant, in ZS05 the percentage of saturated FFAs further increased to $59 \%$, indicating the possible redox changes between NAD and NADP redox couples.

\section{Increased provision of acetyl-CoA and NADPH improved FFA production}

Plasmid pGapN was transformed into PDH1 and PDH2, yielding PDH3 and PDH6, respectively. As shown in Fig. 4, the FFA production in PDH3 and PDH6 increased to 534.0 and $797.6 \mathrm{mg} / \mathrm{L}$, respectively. Also, glycerol accumulation levels in PDH3 and PDH6 reduced to 0.93 and $0.58 \mathrm{~g} / \mathrm{L}$, respectively. The decrease in glycerol level and increase in FFA titer in both PDH3 and PDH6 were similar with those observed in ZS02.

Similarly, when GPD1 and GPD2 were deleted in PDH1 and PDH2, the resulting strains PDH4 and PDH7 exhibited decreased growth rates, but increased FFA production to 664.0 and $840.5 \mathrm{mg} / \mathrm{L}$, respectively (Fig. 4). Finally, when GapN was introduced into PDH4 and PDH7, yielding PDH5 and PDH8, the impaired cell growth improved, whereas the FFA production decreased to 614.0 and $715.3 \mathrm{mg} / \mathrm{L}$, respectively (Fig. 4). The improved cell growth and decreased FFA titer upon GapN expression indicated a possible competition for the supply of the precursors and redox factors. Previous studies with heterologous acetyl-CoA pathways suggested that the ACL pathway is promising for production of fatty acid and their derivatives $[6,7,10,11,14]$. ACL expression with enhanced fatty acid synthesis resulted in $81 \%$ higher FFAs than YJZ08 $[6,7]$, and in this study the titer of PDH7 was $83.2 \%$ higher compared to YJZ08, similar with ACL expression combined with other engineering efforts.

Regarding the distribution of saturated and unsaturated FFAs (C16 and C18) of engineered PDH strains, it was found that PDH expression significantly increased the ratios of unsaturated FFAs (Fig. 5a), even with efforts for redox cofactor rebalance. These changes might be because of the PDH complex with excess NADH produced and its consistent activity under high $\mathrm{NADH} /$ $\mathrm{NAD}^{+}$ratios. The $\mathrm{PDH}$ complex may be a potential 


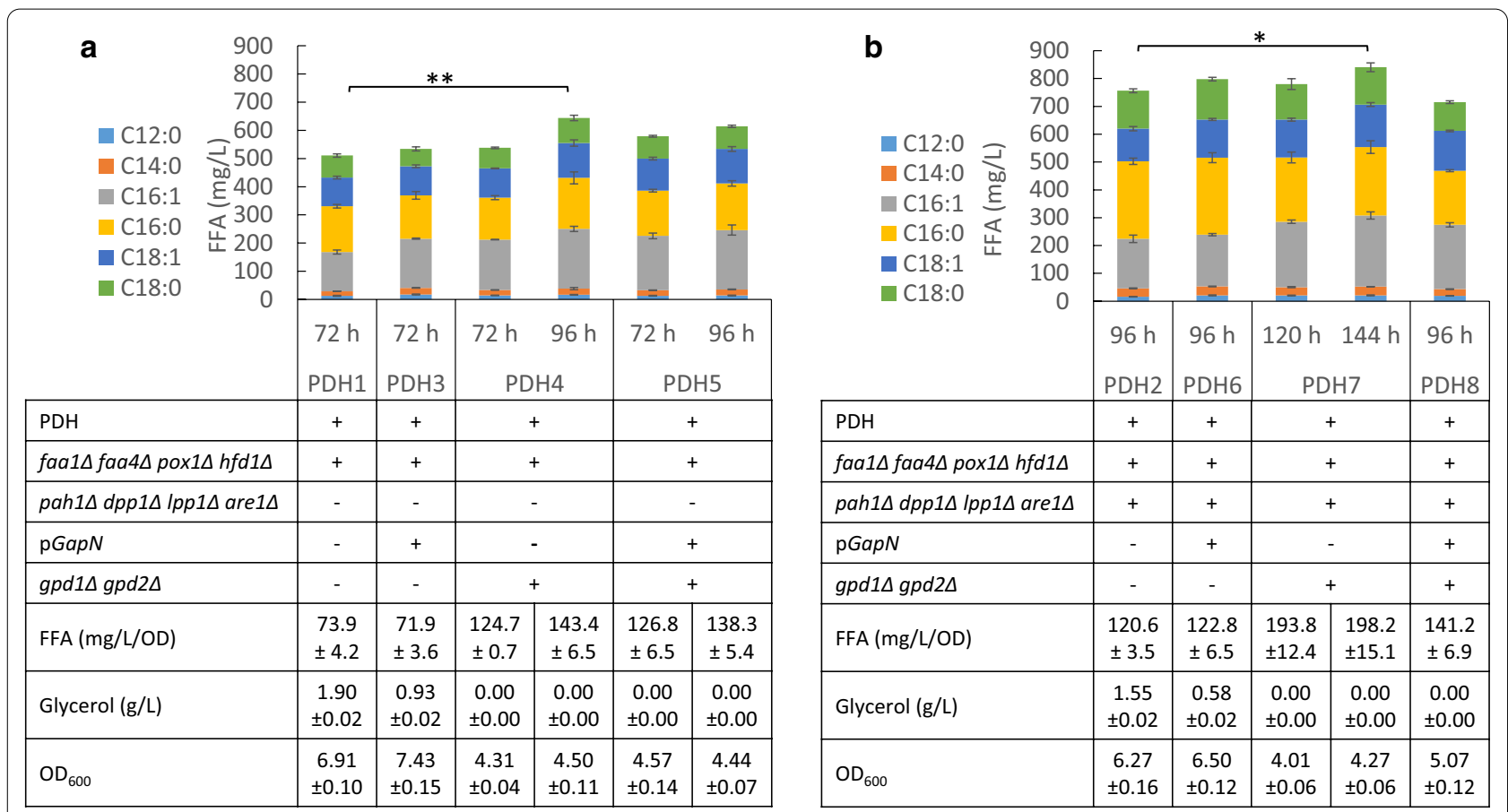

Fig. 4 FFA production with cytosolic $\mathrm{PDH}$ and cofactor engineering. All data represent the mean $\pm \mathrm{SD}$ of biological triplicates

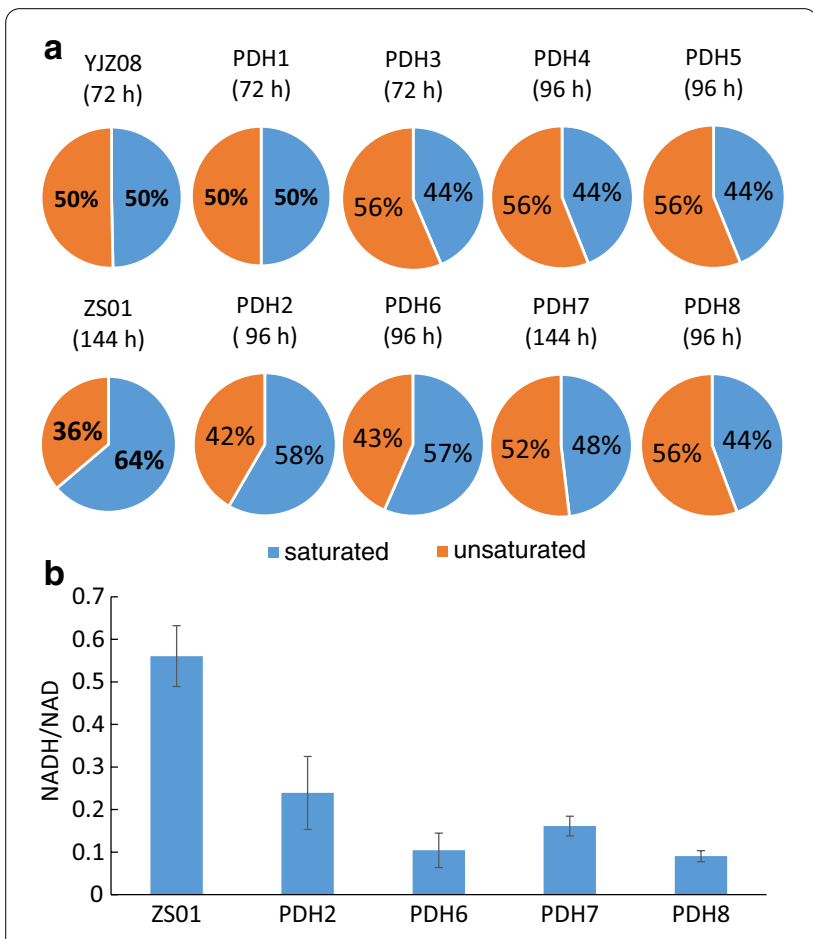

Fig. 5 Distribution of saturated and unsaturated FFAs (C16 and C18) in engineered strains. All data represent the mean \pm SD of biological triplicates target to increase the unsaturation of the FFAs and their derivatives, besides manipulations on Delta-9 fatty acid desaturase Ole1 reported to control the fatty acid saturation in previous studies $[40,41]$.

The intracellular NADH/NAD ${ }^{+}$ratios of the engineered PDH strains were lower than that of ZS01 (Fig. 5b), suggesting the PDH complex alleviated NADH burden, as we conferred from cell growth and FFA production. GapN expression could further relieve the $\mathrm{NADH}$ burden, as the NADH/NAD ${ }^{+}$ratio of PDH6 and PDH8 was lower than that of $\mathrm{PDH} 2$, and the ratio of PDH8 lower than PDH7.

\section{Conclusions}

The expression of the PDH complex from E. faecalis in the yeast cytosol enabled efficient acetyl-CoA provision with low ATP cost, and improved FFA production in the titer, productivity and rate. Rebalancing NAD and NADP redox couples further improved FFA production in PDH expressing strains. Briefly, the GapN expression seemed to increase FFA production via the growth improvement, and GPD deletion resulted in dramatically decreased cell growth probably due to the high cytosolic NADH burden. The strain PDH7 with PDH expression and GPD deletion could achieve a similarly high level of FFA production as other best acetyl-CoA producing pathways, and could potentially engineered for better performances by enhancing fatty acid synthesis and fine-tuning carbon 
metabolism. Moreover, the increase in FFA unsaturation in PDH strains suggested that the PDH complex can be further engineered for the production of unsaturated fatty acids and their derivatives.

\section{Materials and methods}

\section{Construction of plasmids and strains}

The plasmids, strains, and primers used and constructed in this study are listed in Additional file 2: Dataset S1. All yeast strains were constructed using GTR-CRISPR system as described in [8]. For gene integration and deletion, donor DNA containing 50 bp of homology arms and Cas9 plasmid containing gRNA targeting sequence were co-transformed using the electroporation method as described in [42].

Six genes for PDH pathway from Enterococcus faecalis [16] were synthesized by Sangon Company (Shanghai, China) and then cloned in pUC57 vectors, yielding plasmids pPdhB, pPdhA, pLpd, pAceF, pLplA and pLplA2, repectively. $l p l A$ and $l p l A 2$ were cloned using Golden Gate into the vector pAMPori with primers PP1-5, yielding pLplA_A2. These six genes were integrated into two sites XI-3 and XII-5 as described in [28]. lplA and lplA2 were integrated at XI-3 using the Cas9 plasmid pCas9 XI-3.3 and donor DNA amplified from pLplA_A2 with primers PP6 and PP7. $p d h B, p d h A, l p d$ and aceF were integrated at the XII- 5 site simultaneously using pCas9_ XII-5 and donor DNA amplified from the corresponding plasmids with primers PP8-15.

Codon-optimized GapN from Streptococcus mutans [39] was synthesized by Sangon Company (Shanghai, China) and cloned in pAMPori vector using Golden Gate assembly with primers PP16-23, yield pGapN. GapN was then integrated into XI-1 using pCas9_XI-1 and donor DNA amplified with primers PP24 and PP25 from pGapN.

GPD2 was deleted using pCas9_gpd2 and donor DNA amplified with primers PP26 and PP27. GPD1 was deleted using pCas9_gpd1 and donor DNA amplified with primers PP28 and PP29. PAH1, DPP1, LPP1 and ARE1 were simultaneously deleted using pCas9_PDLA and donor DNA fragments with primers PP30-PP37. ZWF1 was deleted using pCas9_zwf1 and donor DNA amplified with primers PP38 and PP39. ALD6 was deleted using pCas9_ald6 and donor DNA amplified with primers PP40 and PP41. LPD was deleted using pCas9_lpd and donor DNA amplified with primer PP42 and PP43.

All Cas9 plasmids containing gRNAs for target genes and were constructed using Golden Gate assembly. Take pCas9_gpd2 as an example, PCR fragments amplified from PScURA with primer PP47, containing 20 bp gRNA targeting sequence, were assembled into pCas plasmid [8].

\section{Media and cultivation conditions}

E. coli strains were cultured at $37^{\circ} \mathrm{C}$ in LB medium, composed of $5 \mathrm{~g} / \mathrm{L}$ yeast extract, $10 \mathrm{~g} / \mathrm{L}$ peptone, and $10 \mathrm{~g} / \mathrm{L}$ $\mathrm{NaCl}$, and $80 \mathrm{mg} / \mathrm{mL}$ ampicillin was added when needed.

Yeast strains were cultured either in YPD medium composed of $10 \mathrm{~g} / \mathrm{L}$ yeast extract, $20 \mathrm{~g} / \mathrm{L}$ peptone and $20 \mathrm{~g} / \mathrm{L}$ glucose, or SC-URA medium for the selection of strains containing URA3-based plasmids, composed of $5 \mathrm{~g} / \mathrm{L}$ $\left(\mathrm{NH}_{4}\right)_{2} \mathrm{SO}_{4}, 1.7 \mathrm{~g} / \mathrm{L}$ yeast nitrogen base without amino acids, $1.914 \mathrm{~g} / \mathrm{L}$ amino acid mixture without uracil, and $20 \mathrm{~g} / \mathrm{L}$ glucose. 5-FOA plates were used to lose URA3based plasmid, composed of $1.7 \mathrm{~g} / \mathrm{L}$ yeast nitrogen base without amino acids, $5 \mathrm{~g} / \mathrm{L}\left(\mathrm{NH}_{4}\right)_{2} \mathrm{SO}_{4}, 1.914 \mathrm{~g} / \mathrm{L}$ amino acid mixture without uracil, $0.05 \mathrm{~g} / \mathrm{L}$ uracil, $1 \mathrm{~g} / \mathrm{L}$ 5-FOA, $25 \mathrm{~g} / \mathrm{L}$ agar. All solid plates contained $15 \mathrm{~g} / \mathrm{L}$ agar except for 5-FOA plates.

Minimal medium was used for cultivation in shake flasks for FFA production, composed of $5 \mathrm{~g} / \mathrm{L}\left(\mathrm{NH}_{4}\right)_{2} \mathrm{SO}_{4}$, $14.4 \mathrm{~g} / \mathrm{L} \mathrm{KH_{2 }} \mathrm{PO}_{4}, 0.5 \mathrm{~g} / \mathrm{L} \mathrm{MgSO}_{4} \cdot 7 \mathrm{H}_{2} \mathrm{O}$, trace metal solution and vitamin solution and $20 \mathrm{~g} / \mathrm{L}$ glucose, and $40 \mathrm{mg} / \mathrm{L}$ histidine or $60 \mathrm{mg} / \mathrm{L}$ uracil was added when needed. For all PDH strains, minimal medium was supplemented with $100 \mathrm{mg} / \mathrm{mL}$ lipoic acid.

Yeast strains were cultured at $30{ }^{\circ} \mathrm{C}$. For shake flask cultivation, yeast cells were precultured in falcon tubes with $5 \mathrm{~mL}$ minimal medium for $24 \mathrm{~h}$, and then inoculated into $100 \mathrm{~mL}$ shake flasks with $30 \mathrm{~mL}$ medium with initial $\mathrm{OD}_{600}$ of 0.1 .

\section{Measurement of biomass and extracellular metabolites}

Biomass was measured by optical density at $600 \mathrm{~nm}$ $\left(\mathrm{OD}_{600}\right)$ with a GENESYS 30 Visible Spectrophotometer (Thermo Electron Scientific, Madison, USA).

Extracellular metabolites including glucose, ethanol and glycerol were measured by HPLC (Shimadzu LC-20AT, Japan) equipped with RID and UV detectors at $210 \mathrm{~nm}$, using $5 \mathrm{mM} \mathrm{H}_{2} \mathrm{SO}_{4}$ as eluent and Aminex HPX-87H column (Bio-Rad) at $65^{\circ} \mathrm{C}$ with a flow rate of $0.6 \mathrm{~mL} / \mathrm{min}$. The injection volume was $10 \mu \mathrm{L}$. A mixture composed of $20 \mathrm{~g} / \mathrm{L}$ glucose, $15 \mathrm{~g} / \mathrm{L}$ ethanol, $0.5 \mathrm{~g} / \mathrm{L}$ pyruvate, $0.5 \mathrm{~g} / \mathrm{L}$ succinate, $2 \mathrm{~g} / \mathrm{L}$ glycerol $2 \mathrm{~g} / \mathrm{L}$ acetate was diluted into 6 gradients with eluent and used as HPLC standards.

\section{Extraction, measurement and analysis of FFAs}

Total FFAs including extracellular and intracellular FFAs were extracted and measured as described in [6]. Specifically, $200 \mu \mathrm{L}$ of cell culture or diluted cell culture was transferred into a glass vial, and $10 \mu \mathrm{L} 40 \%$ tetrabutylammonium hydroxide and $200 \mu \mathrm{L}$ methylation reagent was added immediately. Methylation reagent contained $200 \mathrm{mM}$ methyl iodide as methyl donor and $12.5 \mathrm{mg} / \mathrm{L}$ pentadecanoic acid as an internal standard. 
The mixture was shaken for $30 \mathrm{~min}$, and then centrifuged at $5000 \mathrm{~g}$ for $3 \mathrm{~min}$. $100 \mu \mathrm{L}$ of dichloromethane layer with extracted methyl esters was transferred into a GC vial, evaporated $3 \mathrm{~h}$, and resuspended in $100 \mu \mathrm{L}$ hexane. Then samples were analyzed by GC-MS (QP2020, Shimadzu, Japan) with a DB-5MS column (30 $\mathrm{m} \times 0.250 \mathrm{~mm} \times 0.25 \mu \mathrm{m}$, Agilent). The program was set as follows: $40{ }^{\circ} \mathrm{C} 2 \mathrm{~min}$; ramp to $130{ }^{\circ} \mathrm{C}$ at $5{ }^{\circ} \mathrm{C} /$ min; raise to $280{ }^{\circ} \mathrm{C}$ at $10{ }^{\circ} \mathrm{C} / \mathrm{min} ; 280{ }^{\circ} \mathrm{C} 3 \mathrm{~min}$. The temperatures of the inlet, mass transfer line and ion source were set at $280,300,230{ }^{\circ} \mathrm{C}$, respectively. Flow rate of carrier gas was $3.0 \mathrm{~mL} / \mathrm{min}$ and the injection volume was $1 \mu \mathrm{L}$. The data were collected in the full inspection mode $(50-650 \mathrm{~m} / \mathrm{z})$, analyzed using GCMS solution 4.4 software and quantified with a standard curve. The standard curve was calibrated using methyl ester standards (F.A.M.E mix, Sigma) for even-chain fatty acids (C8-C22) and pentadecanoic acid (C15) with 6 diluted gradients.

Samples for FFA analysis were harvested at different time points due to varied strain growth. The highest FFA titers were achieved when ethanol was completely consumed. Statistical analysis was performed using one-tailed Student's t test using Microsoft Excel.

\section{Quantification of intracellular NADH/NAD ${ }^{+}$}

Cells were grown to mid-log phase and harvested by centrifugation at $11000 \mathrm{~g}$ for $1 \mathrm{~min}$. Cell pellets were suspended with $400 \mu \mathrm{L}$ of extraction buffer from the $\mathrm{NADH} / \mathrm{NAD}^{+}$Quantitation Kit (Sigma-Aldrich) and transferred to a new tube with $50 \mu \mathrm{L}$ of acid-washed glass beads, and kept on dry ice for $10 \mathrm{~min}$. Cells were then lysed with a Fastprep homogenizer and immediately put on ice for $2-3 \mathrm{~min}$, and centrifuged at 17 $000 \mathrm{~g}$ for $10 \mathrm{~min}$. The supernatant was filtered using a Pierce protein concentrator $(10 \mathrm{~K}$ MWCO PES, Thermo Scientific) to remove proteins according to the kit instruction. All steps between cell harvest and enzymatic assay were carried out at $0{ }^{\circ} \mathrm{C}$. NADH and $\mathrm{NAD}^{+}$ were measured following the manufacturer's instruction with a micro-plate reader at $450 \mathrm{~nm}$.

\section{Supplementary Information}

The online version contains supplementary material available at https://doi. org/10.1186/s12934-020-01493-z.

Additional file 1: Table S1. Ethanol production in engineered strains. Figure S1. FFA production with phosphoketolase pathway expressed in YJZO8 and PDH1. Figure S2. Mutations in E3 resulted in decreased FFA production.

Additional file 2. Dataset S1. Primers, strains and plasmids used and constructed in this study.
Abbreviations

PDH: Pyruvate dehydrogenase; FFA: Free fatty acid; GPD: NAD ${ }^{+}$-dependent glycerol-3-phosphate dehydrogenase.

\section{Acknowledgements}

The authors thanked for the support from National Natural Science Foundation of China, the Fundamental Research Funds for the Central Universities, State Key Laboratory of Chemical Resource Engineering, the Beijing Advanced Innovation Center for Soft Matter Science and Engineering, Beijing University of Chemical Technology, the Novo Nordisk Foundation, and the Knut and Alice Wallenberg Foundation.

\section{Authors' contributions}

$Y Z, M S, J N$ and $Z L$ designed the research and wrote the paper; $Y Z, M S, N Q$ performed the experiment. $Z \mathrm{~L}$ and JN supervised the research. All authors read and approved the final manuscript.

\section{Funding}

This work was supported by National Natural Science Foundation of China (21908004 and 21808008), the Fundamental Research Funds for the Central Universities (buctrc201801), the Novo Nordisk Foundation (NNF10CC1016517), and the Knut and Alice Wallenberg Foundation.

\section{Availability of data and materials}

All datasets generated and analyzed during this study are included in this published article and its supplementary information files.

\section{Ethics approval and consent to participate}

Not applicable.

\section{Consent for publication}

Not applicable.

\section{Competing interests}

The authors declare no competing interests.

\section{Author details}

${ }^{1}$ Beijing Advanced Innovation Center for Soft Matter Science and Engineering, College of Life Science and Technology, Beijing University of Chemical Technology, No.15 North Third Ring Road East, Chaoyang District, Beijing 100029, People's Republic of China. ${ }^{2}$ Department of Biology and Biological Engineering, Chalmers University of Technology, Gothenburg, Sweden. ${ }^{3}$ Biolnnovation Institute, Ole Maaløes Vej 3, 2200 Copenhagen N, Denmark.

Received: 12 August 2020 Accepted: 3 December 2020

Published online: 10 December 2020

\section{References}

1. Tehlivets $\mathrm{O}$, Scheuringer K, Kohlwein SD. Fatty acid synthesis and elongation in yeast. Biochim Biophys Acta. 2007;1771(3):255-70.

2. Zhang Y, Nielsen J, Liu Z. Metabolic engineering of Saccharomyces cerevisiae for production of fatty acid-derived hydrocarbons. Biotechnol Bioeng. 2018;115(9):2139-47.

3. Hu Y, Zhu Z, Nielsen J, Siewers V. Engineering Saccharomyces cerevisiae cells for production of fatty acid-derived biofuels and chemicals. Open Biol. 2019;9(5):190049.

4. Fernandez-Moya R, Da Silva NA. Engineering Saccharomyces cerevisiae for high-level synthesis of fatty acids and derived products. FEMS Yeast Res. 2017. https://doi.org/10.1093/femsyr/fox071.

5. Runguphan W, Keasling JD. Metabolic engineering of Saccharomyces cerevisiae for production of fatty acid-derived biofuels and chemicals. Metab Eng. 2014;21:103-13.

6. Zhou YJ, Buijs NA, Zhu Z, Qin J, Siewers V, Nielsen J. Production of fatty acid-derived oleochemicals and biofuels by synthetic yeast cell factories. Nat Commun. 2016;7:11709.

7. Yu T, Zhou YJ, Huang M, Liu Q, Pereira R, David F, et al. Reprogramming yeast metabolism from alcoholic fermentation to lipogenesis. Cell. 2018;174(6):1549-58.e14. 
8. Zhang Y, Wang J, Wang Z, Zhang Y, Shi S, Nielsen J, et al. A gRNA-tRNA array for CRISPR-Cas9 based rapid multiplexed genome editing in Saccharomyces cerevisiae. Nat Commun. 2019;10(1):1053.

9. Ferreira R, Teixeira PG, Siewers $V$, Nielsen J. Redirection of lipid flux toward phospholipids in yeast increases fatty acid turnover and secretion. Proc Natl Acad Sci U S A. 2018;115(6):1262-7.

10. Li X, Guo D, Cheng Y, Zhu F, Deng Z, Liu T. Overproduction of fatty acids in engineered Saccharomyces cerevisiae. Biotechnol Bioeng. 2014;111(9):1841-52.

11. Ghosh A, Ando D, Gin J, Runguphan W, Denby C, Wang G, et al. (13)C metabolic flux analysis for systematic metabolic engineering of S. cerevisiae for overproduction of fatty acids. Front Bioeng Biotechnol. 2016;4:76.

12. Tang $X L$, Feng $H X$, Chen WN. Metabolic engineering for enhanced fatty acids synthesis in Saccharomyces cerevisiae. Metab Eng. 2013;16:95-102.

13. Feng $X$, Lian J, Zhao H. Metabolic engineering of Saccharomyces cerevisiae to improve 1-hexadecanol production. Metab Eng. 2015;27:10-9.

14. de Jong BW, Shi S, Siewers V, Nielsen J. Improved production of fatty acid ethyl esters in Saccharomyces cerevisiae through up-regulation of the ethanol degradation pathway and expression of the heterologous phosphoketolase pathway. Microb Cell Fact. 2014;13(1):39.

15. van Rossum HM, Kozak BU, Pronk JT, van Maris AJA. Engineering cytosolic acetyl-coenzyme A supply in Saccharomyces cerevisiae: Pathway stoichiometry, free-energy conservation and redox-cofactor balancing. Metab Eng. 2016;36:99-115.

16. Kozak BU, van Rossum HM, Luttik MA, Akeroyd M, Benjamin KR, Wu L, et al. Engineering acetyl coenzyme a supply: functional expression of a bacterial pyruvate dehydrogenase complex in the cytosol of Saccharomyces cerevisiae. mBio. 2014. https://doi.org/10.1128/mBio.01696-14.

17. Lian J, Zhao H. Functional reconstitution of a pyruvate dehydrogenase in the cytosol of Saccharomyces cerevisiae through lipoylation machinery engineering. ACS Synth Biol. 2016;5(7):689-97.

18. Lian J, Si T, Nair NU, Zhao H. Design and construction of acetyl-CoA overproducing Saccharomyces cerevisiae strains. Metab Eng. 2014;24:139-49.

19. Papapetridis I, van Dijk M, van Maris AJA, Pronk JT. Metabolic engineering strategies for optimizing acetate reduction, ethanol yield and osmotolerance in Saccharomyces cerevisiae. Biotechnol Biofuels. 2017;10:107.

20. Zhang L, Tang Y, Guo ZP, Ding ZY, Shi GY. Improving the ethanol yield by reducing glycerol formation using cofactor regulation in Saccharomyces cerevisiae. Biotechnol Lett. 2011;33(7):1375-80.

21. Bro C, Regenberg B, Forster J, Nielsen J. In silico aided metabolic engineering of Saccharomyces cerevisiae for improved bioethanol production. Metab Eng. 2006;8(2):102-11.

22. Guadalupe Medina V, Almering MJ, van Maris AJ, Pronk JT. Elimination of glycerol production in anaerobic cultures of a Saccharomyces cerevisiae strain engineered to use acetic acid as an electron acceptor. Appl Environ Microbiol. 2010;76(1):190-5.

23. Ansell R, Granath $K$, Hohmann S, Thevelein JM, Adler L. The two isoenzymes for yeast NAD+-dependent glycerol 3-phosphate dehydrogenase encoded by GPD1 and GPD2 have distinct roles in osmoadaptation and redox regulation. EMBO J. 1997;16(9):2179-87.

24. Navarrete C, Nielsen J, Siewers V. Enhanced ethanol production and reduced glycerol formation in fps 1 mutants of Saccharomyces cerevisiae engineered for improved redox balancing. AMB Express. 2014;4(1):86.

25. Wang P-M, Zheng D-Q, Ding R, Chi X-Q, Tao X-L, Min H, et al. Improvement of ethanol production in Saccharomyces cerevisiae by heteroexpression of GAPN and FPS1 deletion. J Chem Technol Biotechnol. 2011;86(9):1205-10.

26. Kocharin K, Chen Y, Siewers V, Nielsen J. Engineering of acetyl-CoA metabolism for the improved production of polyhydroxybutyrate in Saccharomyces cerevisiae. AMB Express. 2012;2(1):52.
27. Chen Y, Bao J, Kim IK, Siewers V, Nielsen J. Coupled incremental precursor and co-factor supply improves 3-hydroxypropionic acid production in Saccharomyces cerevisiae. Metab Eng. 2014;22:104-9.

28. Mikkelsen MD, Buron LD, Salomonsen B, Olsen CE, Hansen BG, Mortensen $U \mathrm{H}$, et al. Microbial production of indolylglucosinolate through engineering of a multi-gene pathway in a versatile yeast expression platform. Metab Eng. 2012;14(2):104-11.

29. Bergman A, Siewers V, Nielsen J, Chen Y. Functional expression and evaluation of heterologous phosphoketolases in Saccharomyces cerevisiae. Amb Express. 2016;6:115.

30. Meadows AL, Hawkins KM, Tsegaye Y, Antipov E, Kim Y, Raetz L, et al. Rewriting yeast central carbon metabolism for industrial isoprenoid production. Nature. 2016;537(7622):694-7.

31. Maaheimo H, Fiaux J, Cakar ZP, Bailey JE, Sauer U, Szyperski T. Central carbon metabolism of Saccharomyces cerevisiae explored by biosynthetic fractional (13)C labeling of common amino acids. Eur J Biochem. 2001;268(8):2464-79.

32. Minard Kl, MCAlister-Henn L. Sources of NADPH in yeast vary with carbon source. J Biol Chem. 2005;280(48):39890-6.

33. Bocanegra JA, Scrutton NS, Perham RN. Creation of an NADP-dependent pyruvate dehydrogenase multienzyme complex by protein engineering. Biochemistry. 1993;32(11):2737-40.

34. Cardenas J, Da Silva NA. Engineering cofactor and transport mechanisms in Saccharomyces cerevisiae for enhanced acetyl-CoA and polyketide biosynthesis. Metab Eng. 2016;36:80-9.

35. Wu J, Wang Z, Duan X, Zhou P, Liu P, Pang Z, et al. Construction of artificial micro-aerobic metabolism for energy- and carbon-efficient synthesis of medium chain fatty acids in Escherichia coli. Metab Eng. 2019;53:1-13.

36. Snoep JL, de Graef MR, Westphal AH, de Kok A, de Teixeira Mattos MJ, Neijssel OM. Differences in sensitivity to NADH of purified pyruvate dehydrogenase complexes of Enterococcus faecalis, Lactococcus lactis, Azotobacter vinelandii and Escherichia coli: implications for their activity in vivo. FEMS Microbiol Lett. 1993;114(3):279-83.

37. van Rossum HM, Kozak BU, Niemeijer MS, Dykstra JC, Luttik MA, Daran $J M$, et al. Requirements for carnitine shuttle-mediated translocation of mitochondrial acetyl moieties to the yeast cytosol. mBio. 2016. https:// doi.org/10.1128/mBio.00520-16.

38. Zhang J, Sonnenschein N, Pihl TP, Pedersen KR, Jensen MK, Keasling JD. Engineering an NADPH/NADP(+) Redox Biosensor in Yeast. ACS Synth Biol. 2016;5(12):1546-56.

39. Boyd DA, Cvitkovitch DG, Hamilton IR. Sequence, expression, and function of the gene for the nonphosphorylating, NADP-dependent glyceraldehyde-3-phosphate dehydrogenase of Streptococcus mutans. J Bacteriol. 1995;177(10):2622-7.

40. Liu Y, Liu Q, Krivoruchko A, Khoomrung S, Nielsen J. Engineering yeast phospholipid metabolism for de novo oleoylethanolamide production. Nat Chem Biol. 2020;16(2):197-205.

41. Bergenholm D, Gossing M, Wei Y, Siewers V, Nielsen J. Modulation of saturation and chain length of fatty acids in Saccharomyces cerevisiae for production of cocoa butter-like lipids. Biotechnol Bioeng. 2018;115(4):932-42.

42. Thompson JR, Register E, Curotto J, Kurtz M, Kelly R. An improved protocol for the preparation of yeast cells for transformation by electroporation. Yeast. 1998;14(6):565-71.

\section{Publisher's Note}

Springer Nature remains neutral with regard to jurisdictional claims in published maps and institutional affiliations. 